\title{
Energy Utilization and Nutrients Removal in Wheat-Weed Ecosystem
}

\author{
Atul Singh*, K.K. Jain and S.D. Upadhyaya \\ Department of Forestry, Jawaharlal Nehru Krishi Vishwa Vidyalaya, \\ Jabalpur 482004 (M.P.), India \\ *Corresponding author
}

\section{Keywords}

Weeds, Crop, Energy, Nutrients, Herbicides

Article Info

Accepted:

17 June 2019

Available Online:

10 July 2019

\section{A B S T R A C T}

The study was initiated to know the energy and nutrients utilization in wheat-weed ecosystem during the year 2016-17 and 2017-18 in 4 years old eucalyptus plantation in Randomized Block Design with three replications. The investigation was carried out at farmer field Village- Majitha Block-Shahpura District- Jabalpur (M.P.). The result revealed that Launaea nudicaulis, Rumax dentatus, Melilotus indicus, Chenopodium album and Phalaris minor are major weed found in experiment area. Launaea nudicaulis, Rumax dentatus, and Melilotus indicus had highest weed dry weight. The significant reduction in dry matter accumulated by these weed species was recorded with the hand weeding and post-emergence application of 2, 4-D @ 0.5 lit ha $^{-1}$ and 2, 4-D @ 0.5 lit ha $^{-1} \mathrm{fb}$ metribuzin @ $0.250 \mathrm{Kg} \mathrm{ha}^{-1}$. The maximum NPK content was observed by Rumax dentatus, Melilotus indicus and Chenopodium album in all the treatment combinations. Under hand weeded plot, the total weeds removed $1.77,0.23,2.73 \mathrm{NPK} \mathrm{kg} \mathrm{ha}{ }^{-1}$ whereas, the maximum nutrients removal was noted in weedy check $28.46,6.27$ and $46.84 \mathrm{~kg} \mathrm{ha}^{-1}$. In wheat-weed ecosystem, the energy utilization by weeds was maximum in weedy check (76.63 lakh k cal ha $\left.{ }^{-1}\right)$ while, the minimum utilization was under hand weeding (6.82 lakh $\mathrm{k} \mathrm{cal} \mathrm{ha}^{-1}$ ) treatment. The energy utilization by the crop was higher with the application of hand weeding ( 267.05 lakh k cal ha $\left.{ }^{-1}\right)$ whereas, the minimum energy was utilized by the crop under weedy check (175.04 lakh k cal ha $\left.{ }^{-1}\right)$. The energy utilized by the wheat seed was maximum ( 81.59 lakh $\left.\mathrm{k} \mathrm{cal} \mathrm{ha}^{-1}\right)$ in hand weeding. The lowest energy utilized by wheat seed was observed under weedy check (49.13 lakh k cal ha ${ }^{-1}$ ).

\section{Introduction}

Agroforestry may be one of the options to increase tree cover upto one third of the total geographical area of the country. The agroforestry (tree + crop) system is more productive and sustainable than pure cropping. India is the first country in the world has implemented the National Agroforestry Policy in 2014, under its
Ministry of Agriculture and Farmers Welfare. Its objective is to expand tree plantation in combination with crops and/or livestock to improve overall productivity, reducing unemployment, generating additional source of income and livelihood support to small landholders. The policy also highlights that agroforestry could be implemented to meet the domestic and industrial requirements of the country for wood and its products. 
Moreover, in this direction, efforts have been made by the farmers and researchers for introducing promising tree based farming systems across the different states in order to attain sustainability (Verma et al., 2017).

Wheat (Triticum aestivum L.) is widely intercropped cereal crop during rabi season (November - April) with Eucalyptus, Poplar, and other fast growing tree species in Northern states of India viz., Uttarakhand, Punjab, Haryana, Uttar Pradesh and Bihar, parts of Central and Eastern states such as Madhya Pradesh, Chhattisgarh and West Bengal. Weed infestation is one of the major factors limiting crop productivity. For realizing full genetic yield potential of the crop, the proper weed control is one of the essential management practices. Weeds not only reduce the yield but also make the harvesting operation difficult. The weeds usually grow faster than crop plants and thus, absorb the available nutrients earlier resulting in lack of nutrients for the growth of the crop plants. Mani (1975) pointed out that the competition for the nutrients in general, and nitrogen in particular in most situation was the serious factor in limiting the crop yield. Uncontrolled weeds are reported to cause upto $66 \%$ reduction in wheat grain yield (Angiras et al., 2008 and Kumar et al., 2011) or even more depending upon the weed density, type of weed flora and duration of infestation.

\section{Materials and Methods}

The field experiment was conducted at the farmer field Village- Majitha Block-Shahpura District- Jabalpur (M.P.) for study the effect of herbicidal treatments in relation to NPK removal and energy utilization by weeds and crop. Wheat crop was intercropped in 4 years old Eucalyptus tereticornis trees with distance of $3 \mathrm{~m} \mathrm{X} 1.5 \mathrm{~m}$. The experiment was laid out in Randomized Block Design with tentreatments under three replications. The treatment combinations consisted of 2, 4-D @ 0.5 lit ha ${ }^{-1}$, Metribuzin @ $0.250 \mathrm{Kg} \mathrm{ha}^{-1}$, Butachlor@1 lit ha ${ }^{-1}$, Clodinafop-propargyl @ $0.140 \mathrm{~kg} \mathrm{ha}^{-1}, 2$,4-D @ 0.5 lit ha ${ }^{-1} \mathrm{fb}$ metribuzin@0.250 Kg ha-1, 2, 4-D@0.5 lit ha $^{-1} f b$ butachlor@1 lit ha ${ }^{-1}$, Metribuzin@ $0.250 \mathrm{Kg} \mathrm{ha}^{-1} \mathrm{fb}$ butachlor@1 lit ha ${ }^{-1}, 2,4-\mathrm{D}$ @ 0.5 lit ha ${ }^{-1}+$ hand weeding at 30 DAS, Hand Weeding at 30 DAS and Weedy check. Wheat variety LOK-1 was sown with $25 \mathrm{~cm}$ row spacing at a depth of $4 \mathrm{~cm}$ from the top of the soil by opening furrows through a Kudal. The weed control treatments and herbicides were applied as post emergent at crop tillering stage i.e. about 30 DAS. The aboveground weed dry matter was also recorded from the above thrown quadrates after cutting weeds from the ground level and then oven dried at $70^{\circ} \mathrm{C}$ and converted to $\mathrm{m}^{2}$.

\section{Nutrient uptake study}

The nitrogen, phosphorus and potassium uptake by weeds and crop was determined at harvest. The weeds were collected, dried, grounded into powder and mixed thoroughly to make a composite sample. The nitrogen was analyzed by Micro-Kjeldal method and phosphorus was analyzed by Phosphomolybdate method (Jackson, 1967), Potash was estimated by Flame-Photometer method as described by Black (1965). Uptake of nutrients by individual weeds, seeds, crop and straw were worked out by multiplying the percentage of nutrients content in dry matter of weeds, seeds and straw per plot.

\section{Energy utilization by weeds and crop}

The energy utilization by weeds and crop was determined after Leith (1965). He reported the energy content of $4.35 \mathrm{k}$ cal per $\mathrm{g}$ weed dry weight and $4.30 \mathrm{k}$ cal per gram in seeds. These values were utilized for converting the dry weights into energy content. 


\section{Results and Discussion}

\section{Weed-Shoot Biomass (dry weight)}

The weed dry weight of various species recorded at the harvest revealed that Launaea nudicaulis, Rumax dentatus, and Melilotus indicus had greater dry weight. The contribution of these weed species was more to the total dry matter production in all the treatments. The significant reduction in dry matter accumulation by these weed species was recorded with the hand weeding 30 DAS and application of 2,4-D and 2, 4-D @ 0.5 lit $\mathrm{ha}^{-1} \mathrm{fb}$ metribuzin @ $0.250 \mathrm{Kg} \mathrm{ha}^{-1}$ for broad leaf weeds. The hand weeding and Clodinafop-propargyl @ $0.140 \mathrm{~kg} \mathrm{ha}^{-1}$ were reducing the dry weight of Phalaris minor. The maximum dry matter accumulation of all the weed species was recorded under weedy check (Table 1). This clearly explained the impact of low light intensities and shaded conditions which favor the growth of weeds more than the crop.

\section{NPK content in weed species}

The major weed flora of the experimental field is presented in Table 2. The NPK content in different weed species revealed that the maximum nitrogen and phosphorus content percentage was noted in Rumax dentatus $(1.67 \%$ and $0.32 \%)$ closely followed by Melilotus indicus (1.47\% and $0.20 \%)$. The minimum nitrogen content was noted in Phalaris minor $(0.18 \%)$. The highest potassium content percentage was noted in Melilotus indicus (2.20\%) followed by Phalaris minor $(2.15 \%)$ and Rumax dentatus (1.94\%). The minimum phosphorus and potassium percentage $(0.17 \%$ and $1.86 \%)$ was noted in Launaea nudicaulis (Table 2).

\section{Crop shoot biomass (straw yield)}

The different weed control treatments caused significant variations on straw production.
Significantly the greater straw production was recorded under hand weeding $43.13 \mathrm{q} \mathrm{ha}^{-1}$. Among different chemical weed control treatment post-emergence application (30 DAS) of Clodinafop-propargyl @ $0.140 \mathrm{~kg}$ $\mathrm{ha}^{-1}$ found higher straw production $(37.94 \mathrm{q}$ $\mathrm{ha}^{-1}$ ) which was at par with Metribuzin @ $0.250 \mathrm{Kg} \mathrm{ha}^{-1}\left(35.97 \mathrm{q} \mathrm{ha}^{-1}\right)$. The lower straw production (31.83 q ha ${ }^{-1}$ ) was found in weedy check (Table 3 ).

\section{Seed yield}

The weed control treatments also caused significant variations on seed yield. The hand weeding showed higher grain production $\left(18.97 \mathrm{q} \mathrm{ha}^{-1}\right)$ than other weed control treatments. Post-emergence application of Clodinafop-propargyl @ $0.140 \quad \mathrm{~kg} \mathrm{ha}^{-1}$ produced the higher grain yield $\left(16.91 \mathrm{q} \mathrm{ha}^{-1}\right)$ as compared to all other treatments. The increased in yield was obviously due to reduced weed stress and increased yield attributes. The lowest grain yield $(12.57 \mathrm{q}$ $\mathrm{ha}^{-1}$ ) observed in weedy check due to greater weed competition stress (Table 3).

\section{NPK removal by weeds}

The NPK removal by different weeds revealed that Launaea nudicaulis, Rumax dentatus, Melilotus indicus, Chenopodium album and Phalaris minor removed greater NPK in all the treatment combinations. Rumax dentatus and Launaea nudicaulis caused greater remove of NPK under weedy check and herbicidal treated plots. Phalaris minor removed less amount of NPK $\mathrm{kg} \mathrm{ha}^{-1}$ (Table 4). The total weeds under hand weeded plots removed 1.77, 0.23, 2.73 NPK $\mathrm{kg} \mathrm{ha}^{-1}$. The plots where weeding was not done i.e. weedy check, the total weeds removed 28.46, 6.27, 46.84 NPK kg ha-1. The use of herbicides helped to the greater extent for utilization of the nutrients by the crop and reduced their removal by the weeds (Table 4 ). 
Table.1 Effect of different weed control treatments on weedy dry weight $\left(\mathrm{g} \mathrm{m}^{2}\right)$ under the shaded conditions Eucalyptus tereticornis based agroforestry system

\begin{tabular}{|c|c|c|c|c|c|c|}
\hline \multicolumn{2}{|r|}{ Treatments } & \multirow{2}{*}{$\begin{array}{c}\text { Phalaris } \\
\text { minor }\end{array}$} & \multirow{2}{*}{$\begin{array}{c}\text { Chenopodium } \\
\text { album }\end{array}$} & \multirow{2}{*}{$\begin{array}{c}\begin{array}{c}\text { Rumax } \\
\text { dentatus }\end{array} \\
2.11 \\
(6.00)\end{array}$} & \multirow{2}{*}{$\begin{array}{c}\begin{array}{c}\text { Launaea } \\
\text { nudicaulis }\end{array} \\
2.72 \\
(8.00)\end{array}$} & \multirow{2}{*}{$\begin{array}{c}\text { Melilotus } \\
\text { indicus }\end{array}$} \\
\hline $\mathbf{T}_{1}$ & 2,4-D@0.5 lit ha ${ }^{-1}$ & & & & & \\
\hline $\mathbf{T}_{2}$ & Metribuzin@0.250 Kg ha ${ }^{-1}$ & $\begin{array}{c}2.36 \\
(5.33)\end{array}$ & $\begin{array}{c}3.10 \\
(9.25)\end{array}$ & $\begin{array}{c}4.15 \\
(17.00)\end{array}$ & $\begin{array}{c}3.71 \\
(13.50)\end{array}$ & $\begin{array}{c}2.86 \\
(7.75)\end{array}$ \\
\hline $\mathbf{T}_{3}$ & Butachlor@1 lit ha ${ }^{-1}$ & $\begin{array}{c}3.37 \\
(11.00)\end{array}$ & $\begin{array}{c}3.43 \\
(11.50)\end{array}$ & $\begin{array}{c}4.51 \\
(20.50)\end{array}$ & $\begin{array}{c}4.69 \\
(22.00)\end{array}$ & $\begin{array}{c}3.00 \\
(8.75) \\
\end{array}$ \\
\hline $\mathbf{T}_{4}$ & $\begin{array}{l}\text { Clodinafop-propargyl @ } \\
0.140 \mathrm{~kg} \mathrm{ha}^{-1}\end{array}$ & $\begin{array}{c}1.69 \\
(3.00)\end{array}$ & $\begin{array}{c}4.03 \\
(16.00)\end{array}$ & $\begin{array}{c}5.23 \\
(27.50)\end{array}$ & $\begin{array}{c}4.44 \\
(19.50)\end{array}$ & $\begin{array}{c}3.05 \\
(9.00)\end{array}$ \\
\hline $\mathbf{T}_{5}$ & $\begin{array}{l}\text { 2,4-D@0.5 lit ha }{ }^{-1} \mathrm{fb} \\
\text { metribuzin@0.250 Kg } \\
\text { ha }^{-1}\end{array}$ & $\begin{array}{c}2.52 \\
(6.00)\end{array}$ & $\begin{array}{c}2.57 \\
(7.25)\end{array}$ & $\begin{array}{c}3.49 \\
(12.00)\end{array}$ & $\begin{array}{c}2.72 \\
(9.00)\end{array}$ & $\begin{array}{c}2.00 \\
(4.00)\end{array}$ \\
\hline $\mathbf{T}_{6}$ & $\begin{array}{l}\text { 2,4-D@0.5 lit ha }{ }^{-1} f b \\
\text { butachlor @ } 1 \text { lit ha }\end{array}$ & $\begin{array}{c}3.00 \\
(8.67)\end{array}$ & $\begin{array}{c}3.33 \\
(10.75)\end{array}$ & $\begin{array}{c}4.29 \\
(18.50)\end{array}$ & $\begin{array}{c}4.12 \\
(16.80)\end{array}$ & $\begin{array}{l}2.77 \\
7.40\end{array}$ \\
\hline $\mathbf{T}_{7}$ & $\begin{array}{l}\text { Metribuzin@0.250 Kg } \\
\text { ha }^{-1} f b \text { butachlor@1 lit } \\
\text { ha }^{-1}\end{array}$ & $\begin{array}{c}2.93 \\
(8.33)\end{array}$ & $\begin{array}{c}3.94 \\
(15.25)\end{array}$ & $\begin{array}{c}5.25 \\
(28.00)\end{array}$ & $\begin{array}{c}3.91 \\
(15.00)\end{array}$ & $\begin{array}{c}3.08 \\
(9.25)\end{array}$ \\
\hline $\mathbf{T}_{8}$ & $\begin{array}{l}\text { 2,4-D@0.5 lit ha }{ }^{-1}+\text { hand } \\
\text { weeding at } 30 \mathrm{DAS}\end{array}$ & $\begin{array}{c}2.72 \\
(7.17)\end{array}$ & $\begin{array}{c}2.24 \\
(6.00)\end{array}$ & $\begin{array}{c}2.85 \\
(10.00)\end{array}$ & $\begin{array}{c}3.27 \\
(12.00)\end{array}$ & $\begin{array}{c}2.71 \\
(7.00)\end{array}$ \\
\hline $\mathbf{T}_{9}$ & $\begin{array}{l}\text { Hand Weeding at } 30 \\
\text { DAS }\end{array}$ & $\begin{array}{c}1.60 \\
(2.67)\end{array}$ & $\begin{array}{c}1.61 \\
(3.00)\end{array}$ & $\begin{array}{c}1.61 \\
(4.00)\end{array}$ & $\begin{array}{c}1.78 \\
(4.00)\end{array}$ & $\begin{array}{c}1.39 \\
(2.00)\end{array}$ \\
\hline $\mathbf{T}_{10}$ & Weedy check & $\begin{array}{c}4.59 \\
(20.67)\end{array}$ & $\begin{array}{c}5.25 \\
(27.50)\end{array}$ & $\begin{array}{c}7.07 \\
(50.00)\end{array}$ & $\begin{array}{c}7.11 \\
(50.00)\end{array}$ & $\begin{array}{c}5.29 \\
(28.00)\end{array}$ \\
\hline & SEm \pm & 0.20 & 0.29 & 0.40 & 0.42 & 0.26 \\
\hline & $\mathrm{CD}(\mathrm{P}=\mathbf{0 . 0 5})$ & 0.58 & 0.82 & 1.14 & 1.18 & 0.74 \\
\hline
\end{tabular}


Table. 2 NPK content of different weed species present in wheat field under Eucalyptus tereticornis based agroforestry system (pooled mean of two year)

\begin{tabular}{|r|l|c|c|c|}
\hline \multirow{2}{*}{ S.No. } & \multirow{2}{*}{ Weed species } & \multicolumn{3}{|c|}{ Nutrient content (\%) } \\
\cline { 3 - 5 } & & N & P & K \\
\hline $\mathbf{1}$ & Phalaris minor & 0.18 & 0.19 & 2.15 \\
\hline $\mathbf{2}$ & Chenopodium album & 1.31 & 0.19 & 1.89 \\
\hline $\mathbf{3}$ & Rumax dentatus & 1.67 & 0.32 & 1.94 \\
\hline $\mathbf{4}$ & Launaea nudicaulis & 1.17 & 0.17 & 1.86 \\
\hline $\mathbf{5}$ & Melilotus indicus & 1.47 & 0.20 & 2.20 \\
\hline
\end{tabular}

Table.3 Effect of different weed control treatments on crop shoot biomass and seed yield ( $\mathrm{q} \mathrm{ha}^{-1}$ ) under Eucalyptus tereticornis based agroforestry system (pooled mean of two year)

\begin{tabular}{|c|c|c|c|}
\hline S.No. & Treatment & $\begin{array}{c}\text { Crop-shoot } \\
\text { biomass }\end{array}$ & Seed yield \\
\hline $\mathbf{T}_{1}$ & 2,4-D @0.5 lit ha-1 & 35.56 & 15.92 \\
\hline $\mathbf{T}_{2}$ & Metribuzin @ $0.250 \mathrm{Kg} \mathrm{ha}^{-1}$ & 35.97 & 16.18 \\
\hline $\mathbf{T}_{3}$ & Butachlor@1 lit ha ${ }^{-1}$ & 32.18 & 13.41 \\
\hline $\mathbf{T}_{4}$ & Clodinafop-propargyl @ $0.140 \mathrm{~kg} \mathrm{ha}^{-1}$ & 37.94 & 16.91 \\
\hline $\mathbf{T}_{5}$ & $\begin{array}{l}\text { 2, 4-D @ } 0.5 \text { lit ha }^{-1} f b \text { metribuzin @ } 0.250 \mathrm{Kg} \\
\mathrm{ha}^{-1}\end{array}$ & 35.73 & 15.54 \\
\hline $\mathbf{T}_{6}$ & 2, 4-D @ 0.5 lit ha $^{-1} f b$ butachlor @ 1 lit ha ${ }^{-1}$ & 34.81 & 14.72 \\
\hline $\mathbf{T}_{7}$ & Metribuzin@0.250 Kg ha ${ }^{-1} f b$ butachlor @ 1 lit ha $^{-1}$ & 32.25 & 14.35 \\
\hline $\mathbf{T}_{8}$ & 2, 4-D @ 0.5 lit ha ${ }^{-1}+$ hand weeding at 30 DAS & 35.39 & 16.62 \\
\hline $\mathbf{T}_{9}$ & Hand Weeding at 30 DAS & 43.13 & 18.97 \\
\hline \multirow[t]{3}{*}{$\mathbf{T}_{10}$} & Weedy check & 31.83 & 12.57 \\
\hline & SEm \pm & 0.79 & 0.33 \\
\hline & $\mathrm{CD}(\mathrm{p}=0.05)$ & 2.23 & 0.93 \\
\hline
\end{tabular}


Table.4 Effect of different weed control treatments on removal of nitrogen, phosphorus and potassium $\left(\mathrm{kg}^{-1}\right)$ by weeds in Eucalyptus tereticornis based agroforestry system (pooled mean of two year)

\begin{tabular}{|c|c|c|c|c|c|c|c|c|c|c|c|c|c|c|c|c|c|c|c|}
\hline \multirow{2}{*}{\multicolumn{2}{|c|}{ Treatments }} & \multicolumn{3}{|c|}{ Phalaris minor } & \multicolumn{3}{|c|}{$\begin{array}{c}\text { Chenopodium } \\
\text { album }\end{array}$} & \multicolumn{3}{|c|}{$\begin{array}{c}\text { Rumax } \\
\text { dentatus }\end{array}$} & \multicolumn{3}{|c|}{$\begin{array}{l}\text { Launaea } \\
\text { nudicaulis }\end{array}$} & \multicolumn{3}{|c|}{$\begin{array}{l}\text { Melilotus } \\
\text { indicus }\end{array}$} & \multicolumn{3}{|c|}{ Total } \\
\hline & & $\mathbf{N}$ & $\mathbf{P}$ & $\mathbf{K}$ & $\mathbf{N}$ & $\mathbf{P}$ & $\mathbf{K}$ & $\mathbf{N}$ & $\mathbf{P}$ & $\mathbf{K}$ & $\mathbf{N}$ & $\mathbf{P}$ & $\mathbf{K}$ & $\mathbf{N}$ & $\mathbf{P}$ & $\mathbf{K}$ & $\mathbf{N}$ & $\mathbf{P}$ & $\mathbf{K}$ \\
\hline$T_{1}$ & 2, $\underline{4-\mathrm{D} @ 0.5}$ lit ha ${ }^{-1}$ & 0.24 & 0.27 & 2.96 & 0.61 & 0.08 & 0.86 & 1.63 & 0.33 & 1.78 & 0.93 & 0.13 & 1.45 & 0.72 & 0.10 & 1.30 & 4.13 & 0.91 & 8.34 \\
\hline $\mathbf{T}_{2}$ & $\begin{array}{l}\text { Metribuzin @ } 0.250 \\
\mathrm{Kg} \mathrm{ha}^{-1}\end{array}$ & 0.10 & 0.10 & 1.08 & 1.15 & 0.17 & 1.66 & 2.72 & 0.59 & 3.05 & 1.57 & 0.20 & 2.42 & 1.12 & 0.15 & 1.76 & 6.66 & 1.21 & 9.96 \\
\hline $\mathbf{T}_{\mathbf{3}}$ & $\begin{array}{l}\text { Butachlor @ } 1 \text { lit } \\
\text { ha }^{-1}\end{array}$ & 0.24 & 23 & 2.66 & 1.48 & 0.25 & 2.09 & 3.41 & 0.74 & 3.73 & 2.67 & 0.46 & 4.00 & 1.13 & 0.20 & 2.08 & 8.94 & 1.89 & 14.56 \\
\hline $\mathbf{T}_{4}$ & $\begin{array}{l}\text { Clodinafop- } \\
\text { propargyl @ } 0.140 \\
\mathrm{~kg} \mathrm{ha}^{-1}\end{array}$ & 0.06 & 0.06 & 0.63 & 2.06 & 0.34 & 3.01 & 4.35 & 0.80 & 5.03 & 2.49 & 0.32 & 3.57 & 1.40 & 0.20 & 1.88 & 10.36 & 1.72 & 14.12 \\
\hline $\mathbf{T}_{5}$ & $\begin{array}{l}\text { 2,4-D @ } 0.5 \text { lit ha }^{-1} \\
f b \text { metribuzin @ } \\
0.250 \mathrm{Kg} \mathrm{ha}^{-1}\end{array}$ & 0.09 & 000 & 1.24 & 0.89 & 0.11 & 1.33 & 2.04 & 0.34 & 2.31 & 1.06 & 0.12 & 1.72 & .55 & 0.06 & 0.85 & 4.62 & 0.72 & 7.44 \\
\hline $\mathbf{T}_{6}$ & $\begin{array}{l}\text { 2,4-D@0.5 lit ha }{ }^{-1} \\
f b \text { butachlor @ } 1 \text { lit } \\
\text { ha }^{-1}\end{array}$ & 0.17 & 0.17 & 1.85 & 1.37 & 0.21 & 2.17 & 3.09 & 0.69 & 3.69 & 1.95 & 0.33 & 3.32 & 1.12 & 0.15 & 1.61 & 7.70 & 1.56 & 12.63 \\
\hline $\mathbf{T}_{7}$ & $\begin{array}{l}\text { Metribuzin @ } 0.250 \\
\mathrm{Kg} \mathrm{ha}^{-1} \mathrm{fb} \text { butachlor } \\
\text { @ } 1 \text { lit ha }^{-1}\end{array}$ & 0.16 & 0.16 & 1.82 & 1.92 & 0.28 & 3.08 & 4.72 & 0.90 & 5.63 & 1.80 & 0.25 & 3.04 & 1.30 & 0.18 & 2.01 & 9.90 & 1.77 & 15.57 \\
\hline $\mathbf{T}_{8}$ & $\begin{array}{l}\text { 2,4-D @ } 0.5 \text { lit ha }^{-1} \\
\text { + hand weeding at } \\
30 \text { DAS }\end{array}$ & 0.11 & 0.12 & 1.45 & 0.69 & 0.09 & 1.05 & 1.58 & 0.25 & 1.72 & 1.33 & 0.18 & 2.06 & 1.04 & 0.12 & 1.33 & 4.75 & 0.75 & 7.60 \\
\hline $\mathbf{T}_{9}$ & $\begin{array}{l}\text { Hand Weeding at } 30 \\
\text { DAS }\end{array}$ & 0.03 & 0.03 & 0.48 & 0.37 & 0.04 & 0.52 & 0.64 & 0.09 & 0.69 & 0.40 & 0.05 & 0.68 & 0.33 & 0.02 & 0.35 & 1.77 & 0.23 & 2.73 \\
\hline$T_{10}$ & Weedy check & 0.62 & 0.57 & 5.55 & 5.88 & 0.94 & 7.68 & 10.31 & 2.52 & 14.77 & 6.41 & 1.41 & 10.37 & 5.25 & 0.83 & 8.47 & 28.46 & 6.27 & 46.84 \\
\hline & SEm \pm & 0.02 & 0.03 & 0.23 & 0.21 & 0.04 & 0.28 & 0.47 & 0.10 & 0.51 & 0.29 & 0.04 & 0.45 & 0.26 & 0.04 & 0.40 & 0.73 & 0.14 & 0.98 \\
\hline & $\mathrm{CD}(\mathrm{P}=0.05)$ & 0.06 & 0.08 & 0.65 & 0.60 & 0.10 & 0.80 & 1.34 & 0.28 & 1.44 & 0.82 & 0.12 & 1.28 & 0.73 & 0.10 & 1.12 & 2.06 & 0.41 & 2.77 \\
\hline
\end{tabular}


Table.5 Energy utilization by weeds, crop and seeds (Lakh k cal ha ${ }^{-1}$ ) in wheat-weed ecosystem under Eucalyptus tereticornis based agroforestry system (pooled mean of two year)

\begin{tabular}{|c|c|c|c|c|}
\hline \multirow[t]{2}{*}{ S. No. } & \multirow[t]{2}{*}{ Treatment } & \multicolumn{3}{|c|}{$\begin{array}{c}\text { Energy utilization } \\
\text { (Lakh k cal /ha) }\end{array}$} \\
\hline & & Weeds & Crop & Seeds \\
\hline $\mathbf{T}_{1}$ & 2,4-D@0.5 lit ha-1 & 17.55 & 221.37 & 68.45 \\
\hline $\mathbf{T}_{2}$ & Metribuzin@0.250 Kg ha ${ }^{-1}$ & 22.98 & 224.17 & 69.48 \\
\hline $\mathbf{T}_{\mathbf{3}}$ & Butachlor@1 lit ha ${ }^{-1}$ & 32.08 & 194.96 & 57.84 \\
\hline $\mathbf{T}_{4}$ & Clodinafop-propargyl @0.140 kg ha ${ }^{-1}$ & 32.63 & 235.86 & 72.70 \\
\hline $\mathbf{T}_{\mathbf{5}}$ & 2,4-D@0.5 lit ha ${ }^{-1} f b$ metribuzin @ $0.250 \mathrm{Kg} \mathrm{ha}^{-1}$ & 16.24 & 220.45 & 66.80 \\
\hline $\mathbf{T}_{6}$ & 2,4-D@0.5 lit ha ${ }^{-1} f b$ butachlor @ 1 lit ha ${ }^{-1}$ & 27.02 & 212.95 & 63.28 \\
\hline $\mathbf{T}_{7}$ & Metribuzin@0.250 Kg ha ${ }^{-1} f b$ butachlor @ 1 lit ha ${ }^{-1}$ & 32.99 & 200.37 & 61.71 \\
\hline $\mathbf{T}_{8}$ & 2,4-D@0.5 lit ha ${ }^{-1}+$ hand weeding at 30 DAS & 18.05 & 221.73 & 69.55 \\
\hline $\mathbf{T}_{9}$ & Hand Weeding at $30 \mathrm{DAS}$ & 6.82 & 267.05 & 81.59 \\
\hline \multirow[t]{3}{*}{$\mathbf{T}_{10}$} & Weedy check & 76.63 & 175.04 & 49.13 \\
\hline & SEm \pm & 2.11 & 5.36 & 1.82 \\
\hline & $\mathrm{CD}(\mathrm{p}=0.05)$ & 5.98 & 15.20 & 5.16 \\
\hline
\end{tabular}

\section{Energy utilization}

In the wheat-weed ecosystem, the energy utilization by the weeds was maximum in weedy check (76.63 lakh $\left.\mathrm{k} \mathrm{cal} \mathrm{ha}^{-1}\right)$. Amongst the different weed control treatments, the minimum utilization was under hand weeding (6.82 lakh $\left.\mathrm{k} \mathrm{cal} \mathrm{ha}^{-1}\right)$. The energy utilization by the crop was higher with the hand weeding (267.05 lakh $\mathrm{k}$ cal ha $\left.{ }^{-1}\right)$. Among chemical weed control treatment energy utilization by the crop was higher with Clodinafoppropargyl @ $0.140 \mathrm{~kg} \mathrm{ha}^{-1}$ followed by Metribuzin@0.250 Kg ha ${ }^{-1}$ and 2,4-D@0.5 lit $\mathrm{ha}^{-1}(235.86,224.17$ and 221.37 lakh k cal $\left.\mathrm{ha}^{-1}\right)$. Whereas, the minimum energy was utilized by the crop under weedy check (175.04 lakh k cal ha ${ }^{-1}$ ).

The effect of different weed control treatments revealed that maximum energy utilization by the wheat seed was maximum (81.59 lakh $\mathrm{k}$ cal ha ${ }^{-1}$ ) in hand weeding.
Whereas, in chemical weed control treatment Clodinafop-propargyl @ $0.140 \mathrm{~kg} \mathrm{ha}^{-1}$ at with Metribuzin@0.250 Kg ha ${ }^{-1}$ and 2, 4-D @ 0.5 lit $\mathrm{ha}^{-1}$ was maximum energy utilization by the wheat seed (72.70, 69.48 and 68.45 lakh $\mathrm{k}$ cal $\left.\mathrm{ha}^{-1}\right)$. The lowest energy utilization by wheat seeds was observed under weedy check (49.13 lakh $\mathrm{k}$ cal lakh $\mathrm{k}$ cal ha ${ }^{-1}$ ) due to increased weed competition and reduced yield attributes (Table 5).

From the above going findings it may be concluded that the impact of low light intensities and shaded conditions of eucalyptus plantation favors the growth of weeds more than the crop. Amongst the different weed species Rumax dentatus and Launaea nudicaulis had the greater dry matter accumulation and also removed the greater NPK from the soil in all the treatments. The maximum crop-shoot biomass and seed yield were recorded with the post-emergence application of Clodinafop-propargyl @ 0.140 
$\mathrm{kg} \mathrm{ha}^{-1}$ at 30 DAS (37.94 and $16.91 \mathrm{q} \mathrm{ha}^{-1}$, respectively) as compared to all other treatments. Similarly the greater energy utilization by the wheat crop and seed were also recorded with the application of same herbicides $\left(235.86,72.70\right.$ lakh $\mathrm{k}$ cal ha $\left.{ }^{-1}\right)$ while, the minimum energy was utilized by the weeds under 2, 4-D @ 0.5 lit ha $^{-1} f b$ metribuzin@0.250 Kg ha ${ }^{-1}$ treated/plot (16.24 lakh $\left.\mathrm{k} \mathrm{cal} \mathrm{ha}{ }^{-1}\right)$. Hand weeding 30 DAS may be used in wheat under shaded conditions of eucalyptus tree to reduce the weed growth and removal of nutrients by the weeds which favor the better crop growth and seed yield.

\section{Acknowledgement}

The author of this manuscript is highly thankful to contact farmer and the authority of the Vishwa Vidyalaya for providing the necessary facilities for conducting the experiment.

\section{References}

Angiras NN, Kumar S, Rana SS and Sharma N. 2008. Standardization of dose and time of application of clodinafoppropargyl to manage weeds in wheat. Himachal Journal of Agricultural Research 34(2): 15-18.
Black CA. 1965. Method of Plant and Soil Analysis part 11. Publishing American Society Agronomy, Madison Wiscorsin, USA. pp: 1367-1373.

Jackson ML. 1967. Soil and Plant Analysis Prentica Hal Inc. England Chifta, New York.

Kumar S, Angiras NN and Rana SS. 2011. Bio-efficacy of clodinafop-propargyl + metsulfuron methyl against complex weed flora in wheat. Indian Journal of Weed Science 43(3\&4): 195-198.

Leith H. 1965. Okologische Fiagestellumgen Beider Unterschung def biogischen Staff Prduction. Qualitas Plant .Materiac Vegetabiles 12:241-246.

Mani VS. 1975. Nutrients drain by weed growth in crop fields. Fert. News 20 (2): 21-27.

Yadav SK., Singh, S.P. and Bhan, V.M. 1985. Performance of herbicides for weed control in groundnut. Indian J. Weed Sci. 15(1): 58-61.

Swaminathan M. 1991. Advanced Text Book on food and nutrition Vol 1 Page 212.

Verma P, Bijalwan A, Manmohan, Dobriyal JR, Swamy SL and Thakur TK. 2017. A paradigm shift in agroforestry practices in Uttar Pradesh. Current Science. 112(3): 509- 516.

\section{How to cite this article:}

Atul Singh, K.K. Jain and Upadhyaya, S.D. 2019. Energy Utilization and Nutrients Removal in Wheat-Weed Ecosystem. Int.J.Curr.Microbiol.App.Sci. 8(07): 2162-2169. doi: https://doi.org/10.20546/ijcmas.2019.807.261 